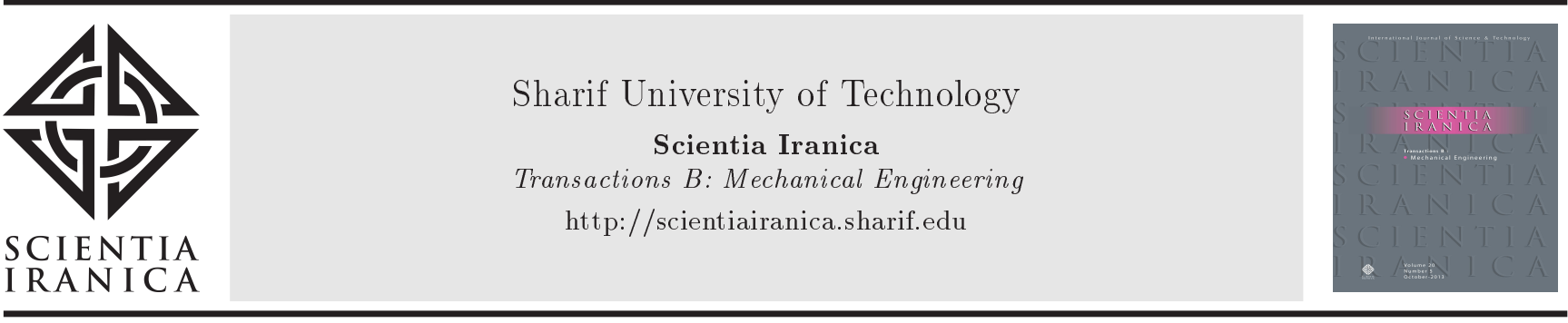

\title{
Experimental investigation of shock wave oscillation on a thin supercritical airfoil
}

\author{
M. Masdari ${ }^{\mathrm{a}, *}$, M. Talebi ${ }^{\mathrm{b}}$, A. Zeinalzadeh ${ }^{\mathrm{b}}$, M.A. Abdi ${ }^{\mathrm{b}}$, and M.R. Soltani ${ }^{\mathrm{b}}$ \\ a. Faculty of New Sciences and Technologies, University of Tehran, Tehran, 1417614418, Iran. \\ b. Department of Aerospace Engineering, Sharif University of Technology, Azadi Ave., Tehran, Zip Code: 1458889694, Iran. \\ Received 29 June 2018; received in revised form 18 September 2018; accepted 24 December 2018
}

\author{
KEYWORDS \\ Pressure distribution; \\ Supercritical airfoil; \\ Transonic regime; \\ Shock wave; \\ Unsteady wake; \\ Buffet phenomenon; \\ Angles of attack; \\ Cross-correlation \\ technique; \\ Schlieren imaging.
}

\begin{abstract}
Experimental results of surface pressure distribution over a thin supercritical airfoil and its wake are presented. All tests were conducted at free stream Mach numbers ranging from 0.27 to 0.85 and at different angles of attacks in a transonic wind tunnel. The model was equipped with static pressure orifices connected to high-frequency pressure transducers. The present paper evaluates variations of shock wave location with both Mach number and angle of attack variation, as well as its interaction with the boundary layer, leading to the buffet phenomenon. Note that, for this thin supercritical airfoil, there exist only a few experimental results regarding surface pressure distributions, corresponding forces and moments, and the shock wave oscillations and its behavior with various flow conditions. The frequency of the shock wave oscillation and unsteady wake behavior at a freestream Mach no. of $M_{\infty}=0.66$ and at different angles of attacks are measured by the cross-correlation technique by means of pressure sensors located on the suction side of the model and via the rake total pressure data that was traversed vertically behind the model, respectively. From the analysis of surface pressure distribution and wake data, drag divergence occurred at a certain angle of attack and at a frequency equal to the shock wave oscillation frequency.

(C) 2020 Sharif University of Technology. All rights reserved.
\end{abstract}

\section{Introduction}

Studies of shock wave interaction with a boundary layer that leads to the shock-induced separation phenomenon and pressure fluctuation at a transonic speed have been carried out as early as the 1950s [1]. Typically, the characteristics of the interaction can be defined by flow characteristics both ahead and downstream of the shock wave, where a separation

\footnotetext{
*. Corresponding author.

E-mail addresses: m.masdari@ut.ac.ir (M. Masdari); msd.talebi@gmail.com (M. Talebi); azeinalzadeh@alum.sharif.edu (A. Zeinalzadeh); ali_abdi@alum.sharif.edu (M.A. Abdi); msoltani@sharif.edu (M.R. Soltani)
}

doi: $10.24200 /$ sci. 2018.51306 .2104 bubble forms. In some circumstances, large-scale fluctuations cause large-scale instability in the entire flow, that is, when the normal shock wave moves over a stupendous portion of a supercritical airfoil, it causes the separation bubble to burst and restructure again periodically. With a constant freestream Mach number, an increase in the angle of attack increases the local Mach number at fixed points upstream of the shock wave. For the small bubble existing at the shock, the disturbance dies out before reaching the trailing edge, where the pressure is practically unaffected by varying the angle of attack. As the bubble grows in size and reaches the trailing edge, it will affect the trailing edge pressure to become divergence. A further increase in the angle of attack results in a greater decrease in the trailing edge pressure, hence a stronger disturbance at the wake. This mixed nature of the flow has important 
consequences in vehicles flying at transonic speeds. In terms of both the structural and the aerodynamic performance, the existence of such an interaction in the flow structure over a wing can provoke serious vibrations and may account for wing or tail destruction, as well as the aileron buzz. This phenomenon is called buffet. In addition, the fluctuation of the lift along the span influences the aircraft flight-envelop for avoiding the limitation on the aircraft flight envelope and fatigue life. Gao et al. [2] studied aerodynamic instability owing to the buffet phenomenon to reduce buffet effects for a wing with active elasticity.

The structure of the separation bubble at the foot of the shock is shown in Figure 1, as taken from Ref. [3].

Lee [4] proposed two types of shock wave boundary layer interaction. In the first type of his model, the supersonic region extends down along the edge of the separation bubble located at the foot of the shock wave, and the boundary layer profile just after the reattachment point in the bubble does not separate from the shock wave. However, in the second model, as seen from Figure 1, the bubble extends to the trailing edge; thereby, the shock wave interacts with the turbulent boundary layer. If the boundary layer is on the verge of separation near the trailing edge when a separation bubble occurs further forward, this bubble will likely disturb the boundary layer profile sufficiently and trigger the rear separation. Buffet onset forms when the separation bubble reaches the trailing edge and bursts. Liu et al. [5] investi- gated the transonic shock buffet instability mechanism by applying a combination of numerical simulations and dynamic mode decomposition over OAT15A and NASA(SC)-0714 supercritical airfoils. They found that the instability of shear layers in the separation zone had the most effect on the onset of unsteadiness of flow, which can burst the separation bubble and detach from the airfoil surface periodically. As a result, the shock traveled along the upper surface. Szubert et al. [6] presented a detailed numerical study of the transonic shock-wave, shear-layer, and wake interactions around a supercritical airfoil at a high Reynolds number. They calculated buffet and Von Karman vorticity frequencies using Power Spectrum Density (PSD) of the wallpressure fluctuations at locations $\frac{x}{c}=0.25,0.45$, and 0.90 on the airfoil surface and at point $\frac{x}{c}=0.25$ and $\frac{y}{c}=0.25$ in the wake, respectively. It should be noted that both spectra on the upper surface of the airfoil are similar in terms of the buffet frequency and its harmonics. On the other hand, a different frequency peak appears, which is generated from the trailing edge and becomes more pronounced in the wake region.

The experimental results of pressure divergence measurement at the trailing edge and the unsteady forces from Polentz et al. [7] and Mabey [8] are common methods for predicting or calculating the buffet onset. Furthermore, Rodrigo et al. [9] presented a new criterion based on the chordwise movement of the aerodynamic center to estimate the transonic buffet onset of transport aircraft. Zhao et al. [10] presented
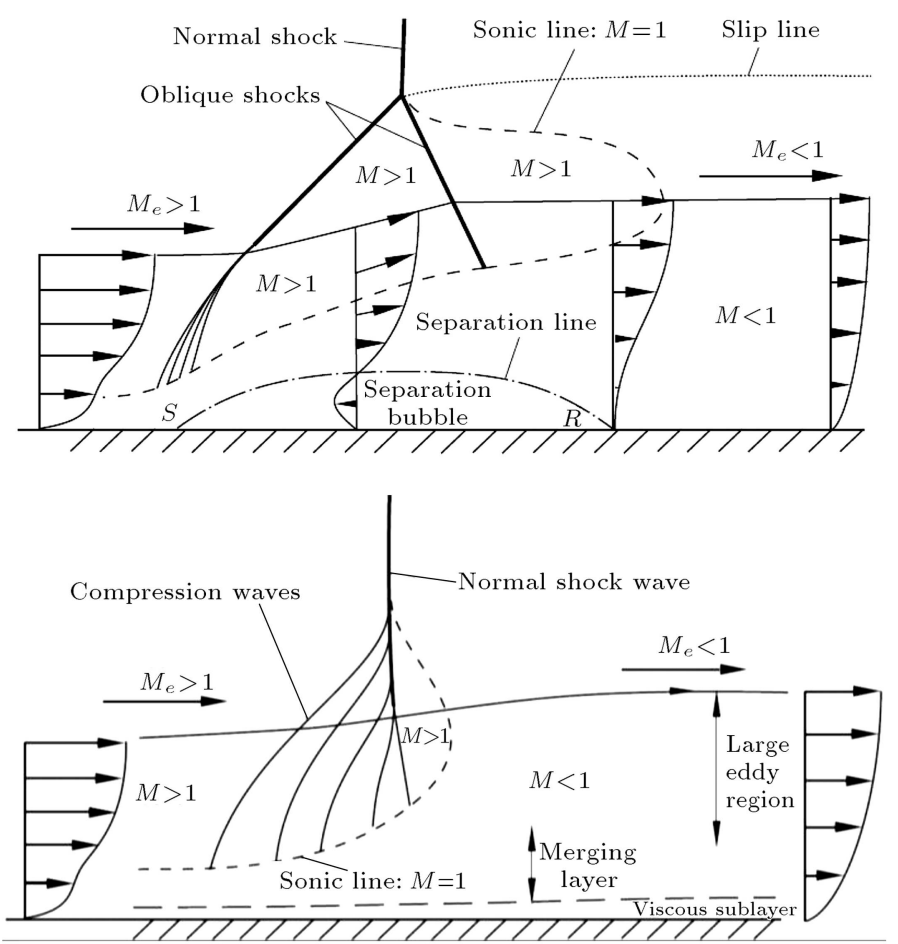

Figure 1. Strong (upper) and weak (lower) shock wave interacting with the boundary layer [3]. 
buffet boundaries at different Mach numbers and angles of attacks for the Sc(2)-714 supercritical airfoil. They indicated that by increasing Mach number, the buffet onset occurred at lower angles of attacks, where RMS of pressure coefficient experienced large fluctuations. The behavior in the shock wave position by increasing Mach number is quite similar to that when the angle of attacks increases. In this case, the free-stream pressure falls as the Mach number increases, and the separation becomes more severe due to the stronger local Mach number ahead of the shock wave. Fernie and Babinsky [11] measured the shock wave oscillation of an NACA0012 airfoil in a quasi-steady flow. They investigated unsteady reduced frequency up to $40 \mathrm{kHz}$ and determined shock location on the upper surface by two alternative methods: pressure trace and image analysis. Pressure trace analysis relies on the examination of the measured $C_{p}$ distributions. In this paper, the location of the shock is based on pressure distribution traces on supercritical airfoil. The location of the shock wave tends to be related to the airfoil profile on which the critical local Mach number also depends, thereby affecting the control of the onset of separation and the rate at which the shock moves over the surface. Pearcey [12] studied shock position on an airfoil, RAE104, at different angles of attacks and various Mach numbers. They demonstrated that the separation bubble influenced the behavior of the shock wave movement via incidence angle. The reason for this phenomenon, back and forward movement of the shock wave with increasing angle of attack, results from the existence of the separation bubble behind the shock at a certain angle of attack. As the angle of attack increases beyond this specified angle, the bubble bursts and will cause a pressure difference across the shock wave. This pressure difference will consequently cause the aforementioned shock wave movement. Note that, in this situation, the pressure coefficient, $C_{p}$, at the foot of the shock is lower than the critical pressure coefficient, $C_{p, c r}$, for this freestream Mach number. A similar phenomenon, shock oscillation, was seen in the present study when the model was set to an angle of attack of 4.9 degrees and at a freestream Mach number of $M_{\infty}=0.66$. The shock wave was found to start oscillating when the model angle of attack increased beyond angle, $\alpha>4.9 \mathrm{deg}$. Lee [13] investigated the shock wave oscillation frequency by applying Fast Fourier Transform (FFT) block size of 256 and a signal duration of $2 \mathrm{sec}$ on the normal force of a $16 \%$ thick supercritical airfoil when its flap was deflected at various angles. A shock wave with a frequency of approximately $50 \mathrm{~Hz}$ appeared when the flap was not deflected $(\beta=0 \mathrm{deg})$, but its intensity increased with increasing $\beta$. To precisely define the conditions for the buffet onset and characterize the properties of the periodic motion from the unsteady surface pressure measurements, Jacquin et al. [14] investigated the shock oscillation over a supercritical airfoil with a relative thickness of $12.3 \%$ in the transonic flow. They used power spectra analysis to derive buffet frequency and spectrogram of pressure signals at two locations on the airfoil to show that the spectral composition of the data is time invariant, which means that the pressure oscillations remain periodic and correlated over the entire extent of the separation region. There are noteworthy studies available about shock oscillation in compressible flow; however, to measure the excitation frequency of oscillating lift on the airfoil in incompressible flow, Tang and Dowell [15] placed NACA0012 at a high angle of attacks well beyond the angle of attack for the onset of stall. An oscillating airfoil at a large angle of attack was used where buffeting occurred at various oscillating frequencies near buffet frequency and several amplitudes. They measured buffet frequency using FFT response of lift coefficient, and found that the oscillating lift on the airfoil occurred when the excitation frequency was sufficiently close to the buffet frequency for stationary airfoil. In recent publications, some researchers claimed that the buffet could be treated as a non-periodic phenomenon if additional body be immersed in the flow shed vortices in the flow over another body. In this case, Kouchi and Yamaguchi [16] experimentally studied transonic buffet on a two-dimensional airfoil with vortex generators using wavelet analysis. They visualized the shock buffet on a two-dimensional transonic airfoil with and without vortex generators by using a fast-framing focusing schlieren imaging. For this case, there was a non-periodic component that was included in the shock oscillation due to the buffet. In addition, some important papers have studied the control of the drag rise of the airfoils under buffet onsets phenomenon, among which Liu and Chichung Yang [17] investigated the flow past a supercritical SC (2)-0714 airfoil at transonic speeds and analyzed the positive effects of microtabs installed on the airfoil used for the alleviation of buffet phenomenon. Furthermore, Tian et al. [18] studied buffet control on a RAE2822 airfoil using two types of the shock control bump. They found that when shock control bump was located downstream of the shock, adverse pressure gradient would decrease. Furthermore, Gao et al. [19] numerically studied transonic buffet suppression by trailing edge flap based on the unsteady Reynolds-averaged Navier-Stokes equations and the Spalart-Allmaras turbulence model for a NACA0012 airfoil. They found that the occurrence of the buffet phenomenon could be completely postponed when the flap deflection is at approximately $50^{\circ}$ phase lead towards the lift response, where the flap deflection is in the reversed phase towards the lift force that affects the buffet flow. There is no quarrel over the fact that the investigation of flow over a supercritical airfoil 


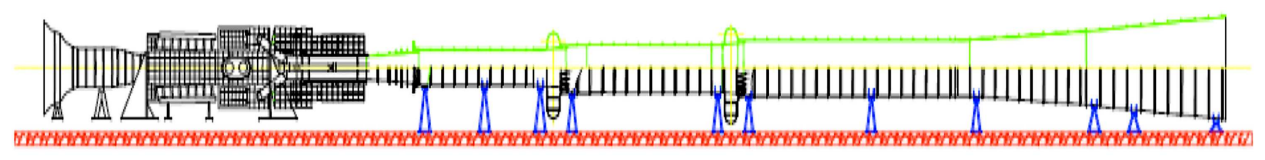

Figure 2. Transonic wind tunnel.

in general and the separation bubble behavior at transonic speeds in particular would constitute complete experimental data for understanding the mechanism of buffet onset. Further, as indicated previously, there exist only a few experimental studies on flow behavior over the present thin supercritical airfoil, which is wildly used in high-performance aircrafts. To fill the existing gap, comprehensive experimental tests were conducted in a transonic wind tunnel using the aforementioned supercritical airfoil. In this case, Golestani et al. [20] captured a shock-wave location using schlieren imaging and presented a new method for detecting the buffet onset using pressure distribution data on the current supercritical airfoil. Additionally, they investigated the effects of wall porosity on the experimental measurements over the same supercritical airfoil [21]. In addition to applying common methods to detect the buffet onset, the specific aim of the current paper is to assess buffet frequency by applying the cross-correlation method to both pressure distribution and wake data behind the model.

\section{Experimental setups}

All experiments were conducted in an upgraded transonic, low-turbulence wind tunnel. Amiri et al. studied a steady flow quality assessment of the transonic wind tunnel [22]. They improved its performance criterion in the transonic regime according to the operational requirements of the various existing transonic wind tunnels. The tunnel is a conventional open-return type with a $60 \times 60 \mathrm{~cm}^{2}$ test section. The tunnel operates at Mach numbers ranging from 0.4 to 2.5 by changing the nozzle profile and the engine RPM. Figure 2 shows a schematic of the wind tunnel, and Figure 3 shows the Mach number distribution along the centerline of the test section at transonic speeds for various Mach numbers.

The upper and lower walls of the test section are perforated at 1\%. Apart from this, there is a chamber beside the walls to control the boundary layer thickness with an additional power plant system. All data were collected via an A/D 64 channels board. Furthermore, data acquisition from high-frequency sensors was done by another 24-bit accuracy A/D board. The sampling frequency was set at $2 \mathrm{kHz}$ and a low-pass filter was designed and implemented in the acquisition program with a cut-off frequency of $200 \mathrm{~Hz}$. All data were acquired at free stream Mach numbers varying from 0.27 to 0.85 . The airfoil model shown in Figure 4(a)

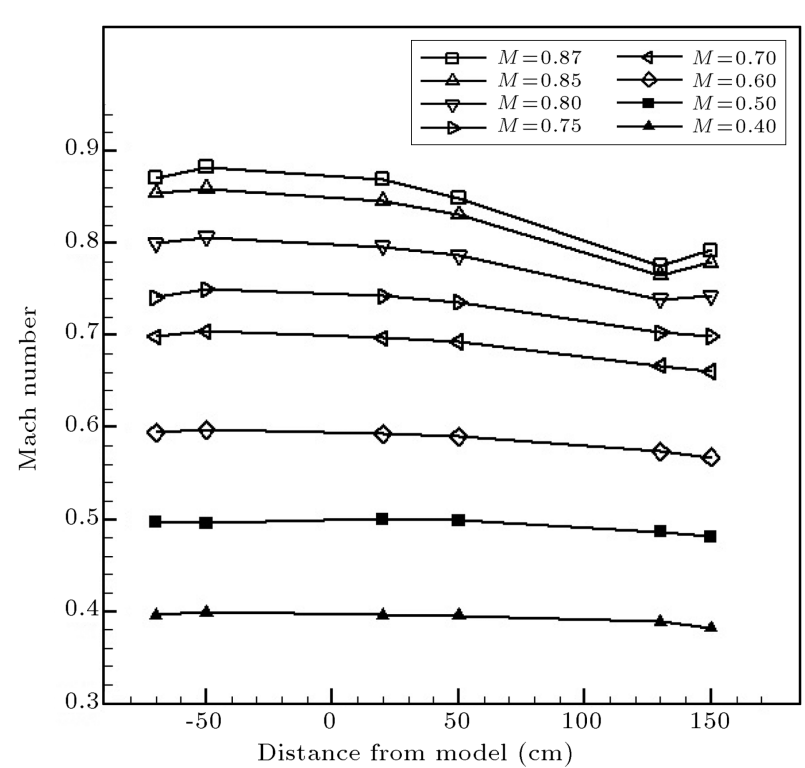

Figure 3. Mach distribution along centerline of transonic wind tunnel test section without model.

had a chord of $20 \mathrm{~cm}$ and a span of $60 \mathrm{~cm}$. The figure shows the location of surface pressure ports over the upper surface of the model. To illustrate the shock-wave oscillations, 17 pressure orifices on the upper surface of the model were connected directly to high-frequency response transducers located inside the model very close to the orifices. Other pressure orifices were connected to the transducers of lower frequency located outside the test section. Figure 4(b) shows a picture of the present model prior to its installation in the wind tunnel.

Figure 5 shows the rake used to measure the wake profile behind the model. Due to some limitations, the farthest distance that the rake could move behind the model was at $30 \%$ of the chord. In these tests, two different rakes were used to measure both static and total pressures in the wake of the model.

\section{Result}

Figure 6 shows surface pressure distribution over the present supercritical airfoil at different Mach numbers and angles of attacks. In Figure 6(a) to (d), it is clearly seen that shock wave moves backward close to the trailing edge by increasing the free stream Mach number at each angle of attack. It is worth mentioning that by increasing $M_{\infty}$, the sonic roof top, as denoted by the dashed lines, decreases, and the shock wave 


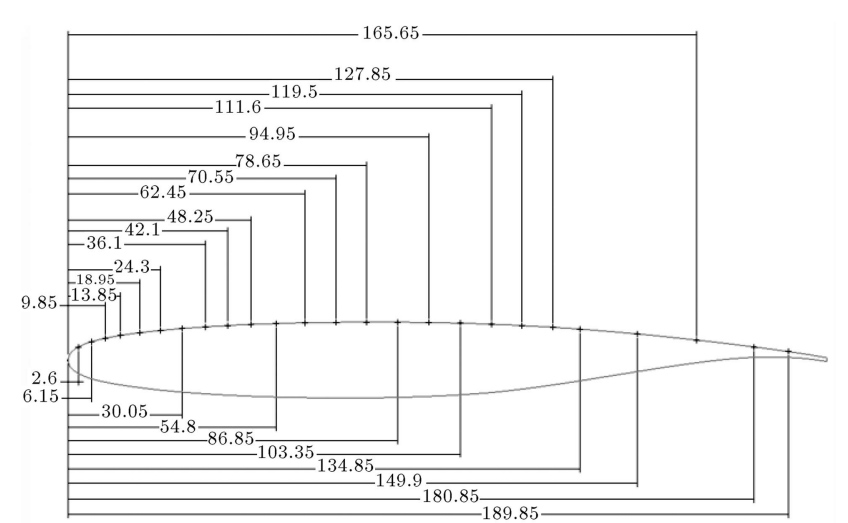

(a) $2 \mathrm{D}$ view of the model

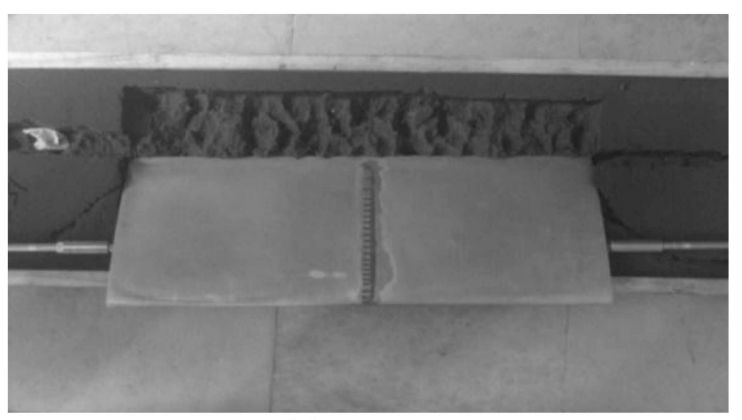

(b) Test model

Figure 4. The supercritical airfoil geometry and location of sensors.

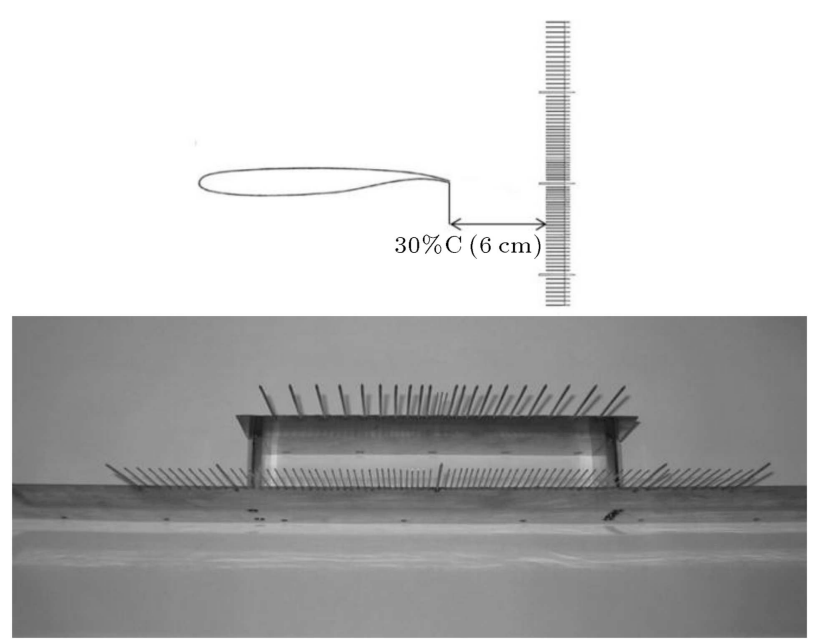

Figure 5. Wake measurement instrument.

moves backward toward the trailing edge. Shock foot locations over the supercritical airfoil for all Mach numbers and angle of attacks tested here are shown in Figure 7. It is seen that the shock wave moves backward towards the trailing edge with the increasing angle of attack at all Mach numbers tested, except for a freestream Mach number of $M_{\infty}=0.66$, whereby the shock at first moves backward and, then, starts to move forward when the incidence angle reaches 4.9 degrees. The buffet boundary at $M_{\infty}=0.66$ can be determined roughly at this angle where the separation bubble at the foot of the shock expands rapidly as the angle of attack increases. According to Figure 7, it appears that the locus of the divergence point of the trailing edge pressure for the present supercritical airfoil occurs at high angles of attacks via Mach number, and this behavior continues until the freestream Mach number reaches 0.71 , where the pressure starts to diverge at lower angles of attack. Figure 8 shows that the separation is first structured at the shock foot on the upper surface when the angle of attack is between 2.8 and 4.9 degrees. Then, pressure falls below the critical pressure (dashed line) and the separation bubble expands rapidly, thereby causing the trailing-edge pressure to diverge (see Figure 9) and the shock halts and moves forward. Note that the pressure coefficient at the trailing edge remains subsonic and no expansion waves are excited from the edge. With this in mind, the shock then moves forward as the angle of attack further increases and no backward motion occurs again. Furthermore, the points at which the separation bubble occurs move forward toward the leading edge.

The onset of shock oscillation on the upper surface of the airfoil is captured here by examining both pressure sensors data located on the upper surface of the model and by the total drag coefficient of the airfoil calculated from the rake transducers. Note that the most common errors are bias errors and precision errors. The bias errors and precision errors were calculated according to the data offsets of the sensors' signals and the Gaussian normal distribution of each sensor, respectively.

Studying the behavior of error bars on pressure distribution diagrams can help predict the buffet onset phenomenon. Shock oscillation causes fluctuation in signals received by highly sensitive pressure sensors, which are located on the upper surface of the airfoil. This phenomenon affects the precision error of the data. Therefore, the local rise in the precision error of the sensors data can account for an unsteady disturbance at a certain frequency. Figure 10 shows that, at $M_{\infty}=0.66$ and for $\alpha=6.7 \mathrm{deg}$, the precision error rises suddenly from $5 \%$ to $30 \%$ of the airfoil chord. For the present supercritical airfoil with $10 \%$ thickness, as shown in Figure 11, at 5.6- and 6.7degree angles of attack, the drag fluctuates at higher amplitudes than it does at the lower angles of attack due to the oscillation of the shock wave on the upper surface. This phenomenon can be studied from time history of the pressure signals (Figure 12) on the upper surface. When the angle of attack increases, the turbulent boundary layer senses high disturbances due to the existence of oscillating shock, resulting in severe fluctuations of all signals behind the shock. Moreover, PSD of the total drag has a large peak near $80 \mathrm{~Hz}$ (Figure 13), while such a large peak does not emerge for other angles of attacks. Accordingly, it can be 


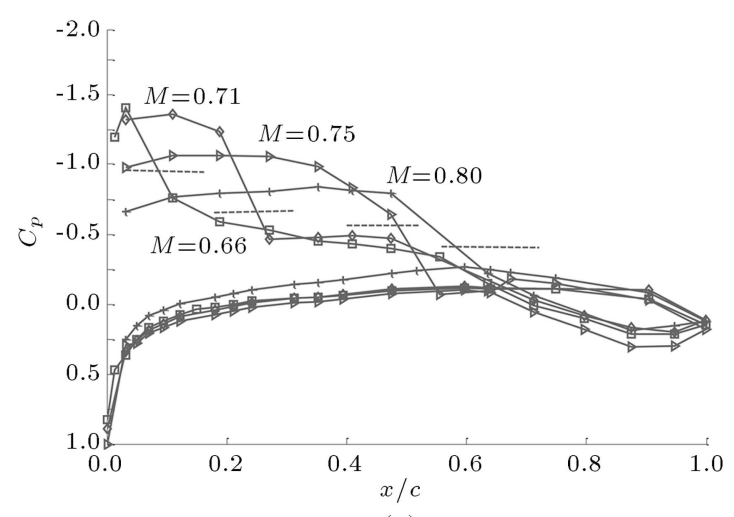

(a)

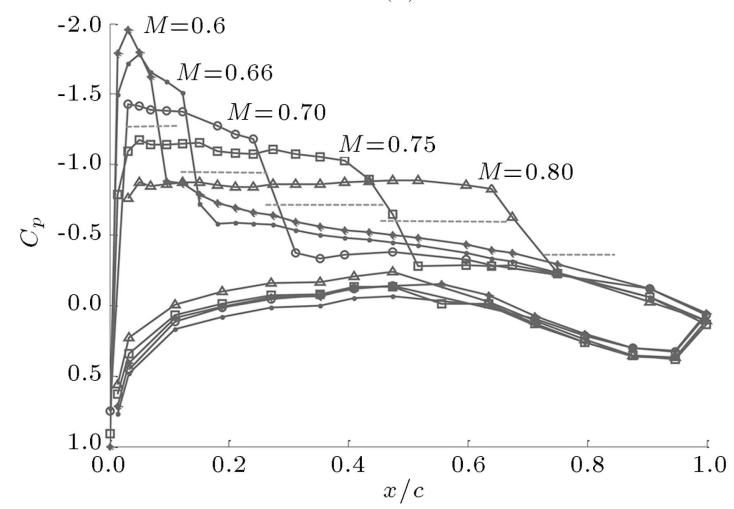

(c)

Figure 6. Pressure distribution over the supercritical airfoil: $\alpha=4.9 \mathrm{deg}$.

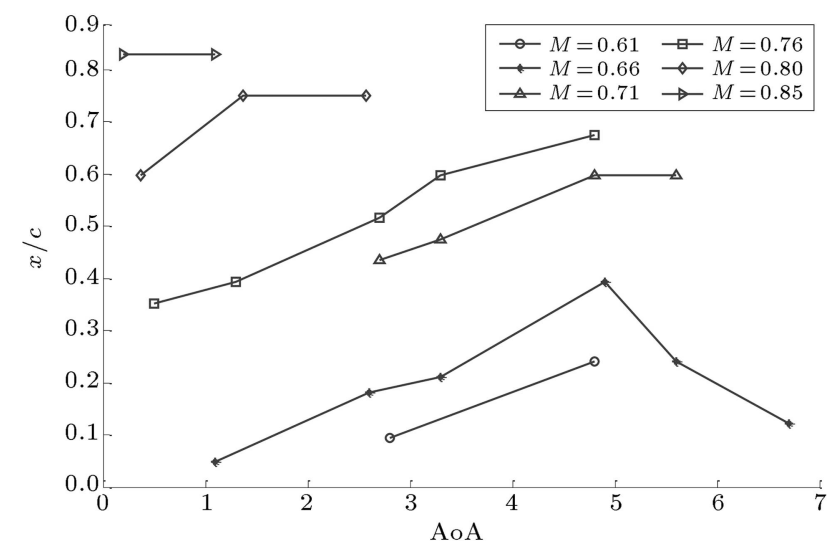

Figure 7. Shock location over the present supercritical airfoil at different angles of attacks and Mach numbers.

implied that the existence of the unsteady shock wave leads to the generation of the main source of unstable frequencies in the wake region.

A sensor near the point of pressure distribution intersection with the sonic line is mostly influenced by the shock-wave oscillation and can be a good choice as a reference signal for cross correlating with other signals. Therefore, the nearest sensor to the foot of the shock wave is selected. The main source of the unstable frequency in flow under the current condition results from the existence of the oscillating shock wave.

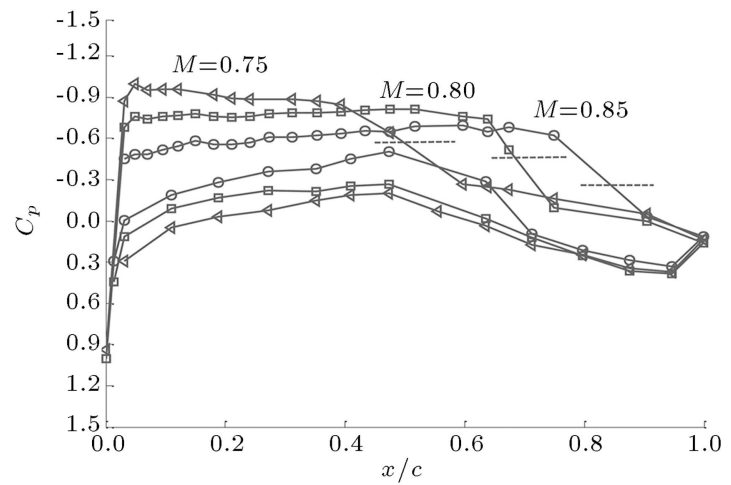

(b)

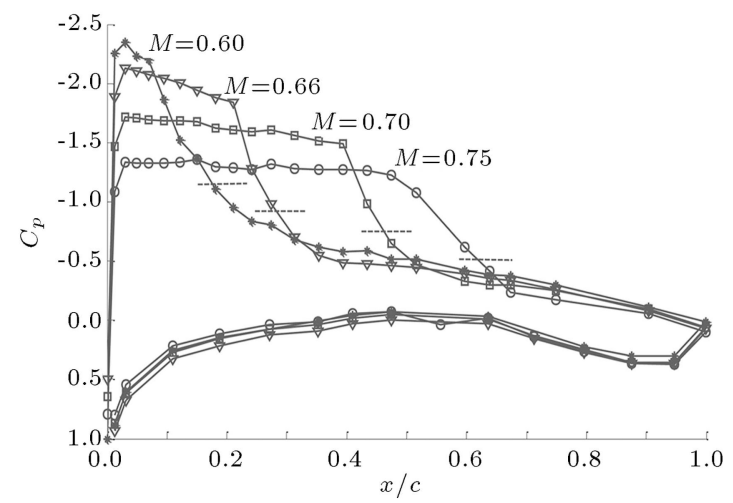

(d)

(a) $\alpha=-4.2 \mathrm{deg}$, (b) $\alpha=1.7 \mathrm{deg}$, (c) $\alpha=2.7 \mathrm{deg}$, and (d)

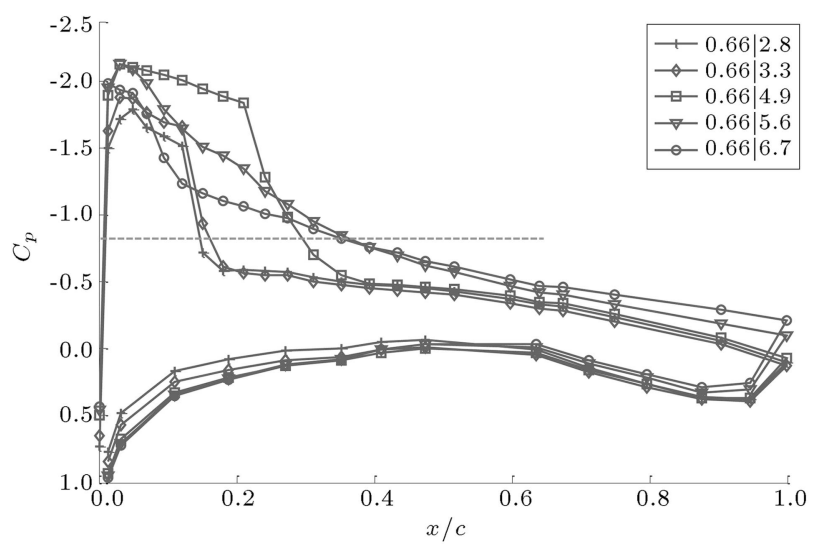

Figure 8. Pressure distribution over the supercritical airfoil at different angles of attack, $M_{\infty}=0.66$.

The oscillation generates a high level of correlation between the sensors located in the oscillating region on the upper surface of the model. In other words, the cross correlation between the reference signal and signals from other sensors located on the upper surface of the model is conveyed such that the shock wave system increases the flow oscillation at other locations with the same frequency. Hence, the frequency of the shock wave oscillation can be obtained through this method.

In the present tests of the $10 \%$ supercritical airfoil, 


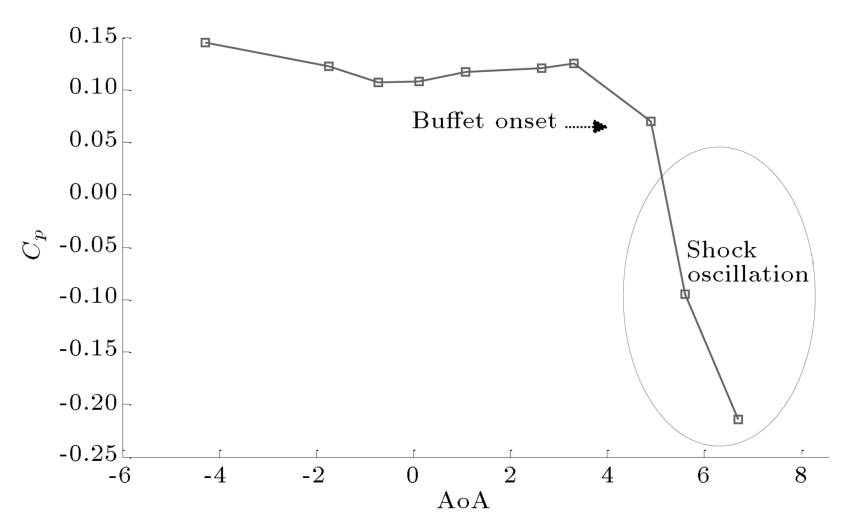

Figure 9. Pressure divergence at the trailing edge of the airfoil at $M_{\infty}=0.66$.

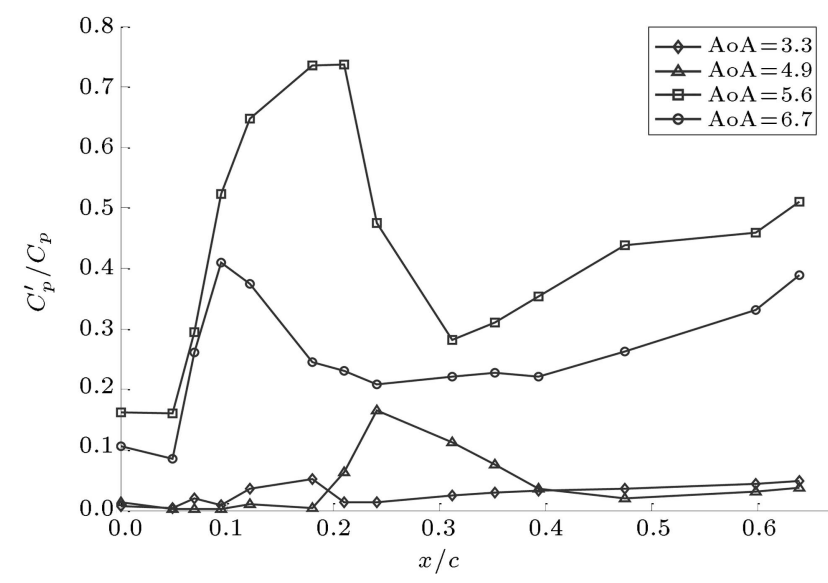

Figure 10. Precision error distribution at $M_{\infty}=0.66$.

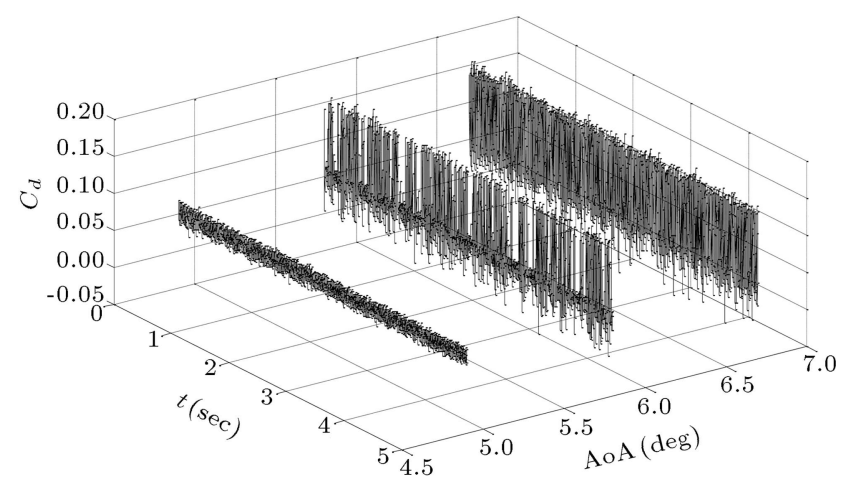

Figure 11. Total drag history at different angles of attack at $M_{\infty}=0.66$.

the shock wave oscillation is not seen clearly at angles of attacks of $\alpha=3.3$ and $\alpha=4.9$ degrees, as seen from Figure 14(a) and (b). However, at an angle of attack of $\alpha=5.6$, Figure 14(c) shows a strong magnitude of cross-correlation between the reference sensor and other sensors located on the upper surface of the airfoil. By increasing the angle of attack to 6.7, as seen in Figure 14(d), the shock wave gets stronger and a sharp rise in the magnitude of the correlation between the sensors is observed. Finally, it can be

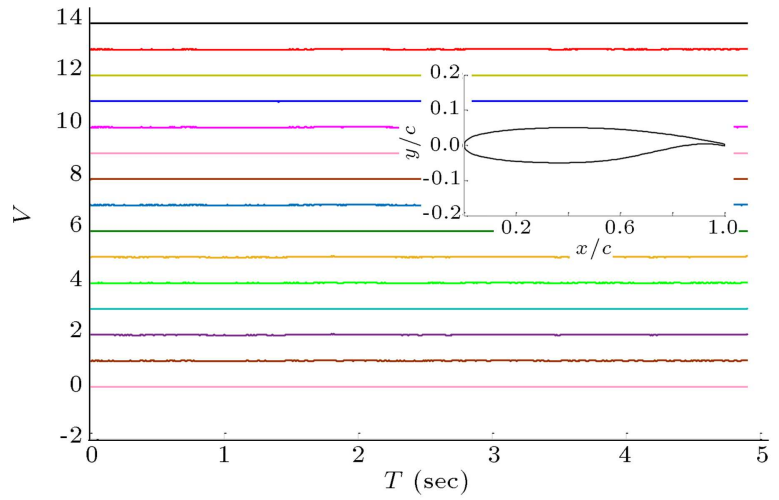

(a)

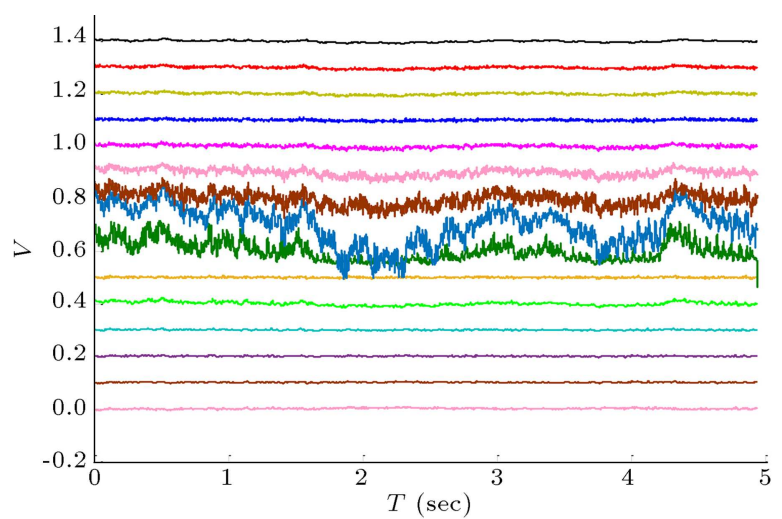

(b)

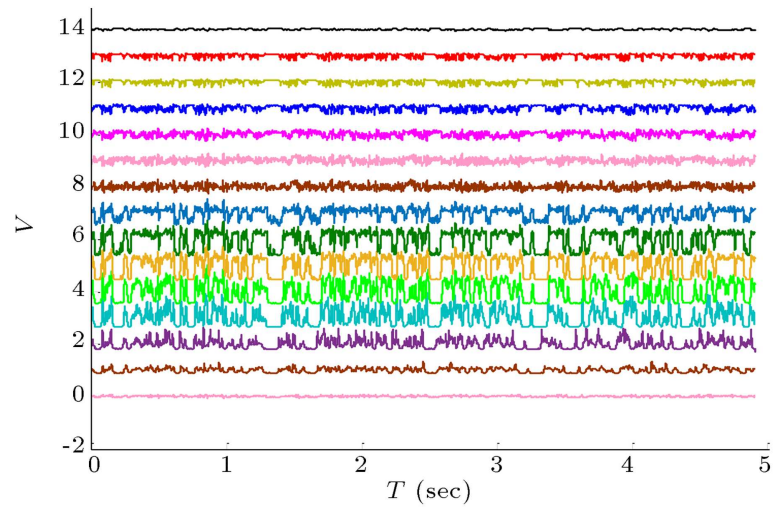

(c)

Figure 12. Time history of the pressure signals on the upper surface of the model at $M_{\infty}=0.66$ : (a) $\alpha=4.9$ $\mathrm{deg}$, (b) $\alpha=5.6 \mathrm{deg}$, and (c) $\alpha=6.7 \mathrm{deg}$.

implied that the buffet onset occurs at $\alpha=4.9$ and the shock wave fluctuates with a frequency of about $80 \mathrm{~Hz}$. After this angle of attack, the shock wave oscillation is set off by the unsteady flow field generated through traveling disturbances upstream on the lower surface that are triggered by the divergence of the trailing edge pressure. This causes periodic oscillations of the circulation of the airfoil, contributing to the oscillation of the circulation in the wake. Therefore, it can be implied that the major frequency of oscillations on the upper surface sensors can be measured by the sensors located in the wake region, too. Similar to the 


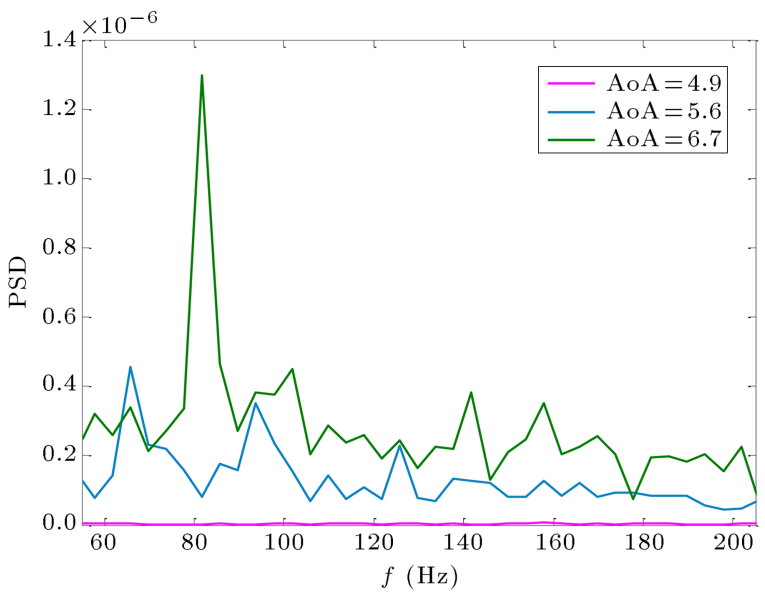

Figure 13. Power spectrum density of total drag.

procedure from which the frequency of shock oscillation was derived, Figure 15(a) shows that there is no strong amplitude at this angle before shock oscillation in the correlation between the sensors located in the wake region. However, Figure 15(b) and 15(c) indicate that when the disturbances are shed in the wake, the same major frequency of oscillation of about $80 \mathrm{~Hz}$ can be detected.

As seen in Figure 16(a)-(c), pressure distribution over the airfoil is plotted. The figures are plotted based

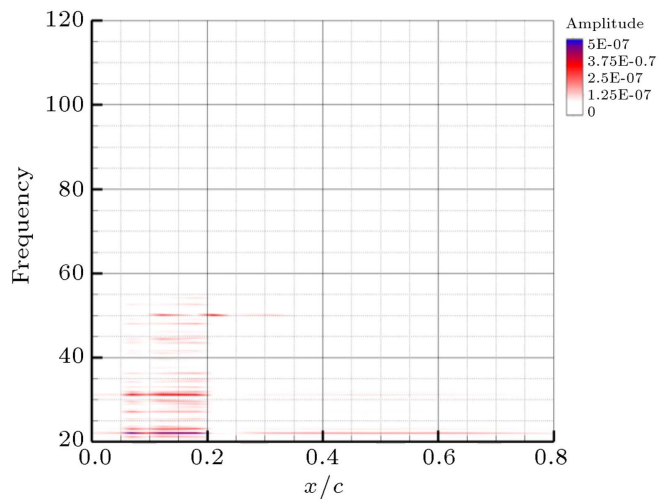

(a)

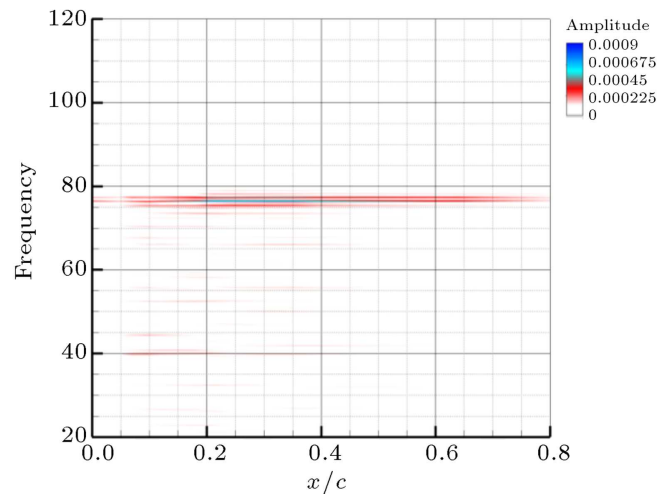

(c) on the most backward and forward positions of the shock wave on the upper surface of the model. In these figures, the boundary of the buffet phenomenon is clearly obvious at an angle of attack of about 4.9 degrees. As seen from these figures, the pressure distributions on the upper and lower surfaces have no fluctuations at $\alpha=4.9$. However, at higher angles of attacks, when the bubble separation at the foot of the shock reaches the trailing edge of the airfoil and bursts, the shock starts to oscillate. Additionally, it is seen that the pressure distribution on the lower side of the airfoil does not show any fluctuation, because this region is not influenced by the shock wave oscillation.

\section{Conclusion}

The effects of free stream Mach number and angles of attack on the shock wave position over the suction side of a thin supercritical airfoil were experimentally investigated. It was observed that with increasing the free stream Mach number, the shock wave moved toward the trailing edge at a fixed angle of attack. Further, at a fixed free stream Mach number, the shock wave moved toward the leading edge with the increasing angle of attack, except that at a certain angle of attack, the pressure coefficient at the trailing edge diverged. This

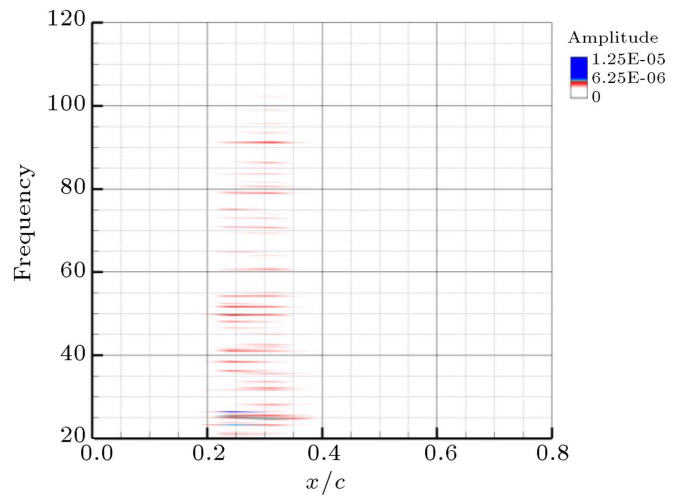

(b)

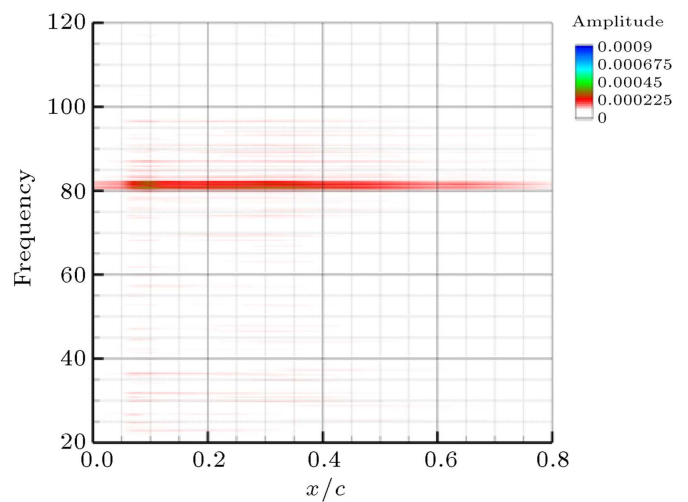

(d)

Figure 14. Cross-correlation diagram of the reference sensor with other sensors signal at $M_{\infty}=0.66:(\mathrm{a}) \alpha=3.3 \mathrm{deg}$, (b) $\alpha=4.9 \mathrm{deg}$, (c) $\alpha=5.6 \mathrm{deg}$, and (d) $\alpha=6.7 \mathrm{deg}$. 


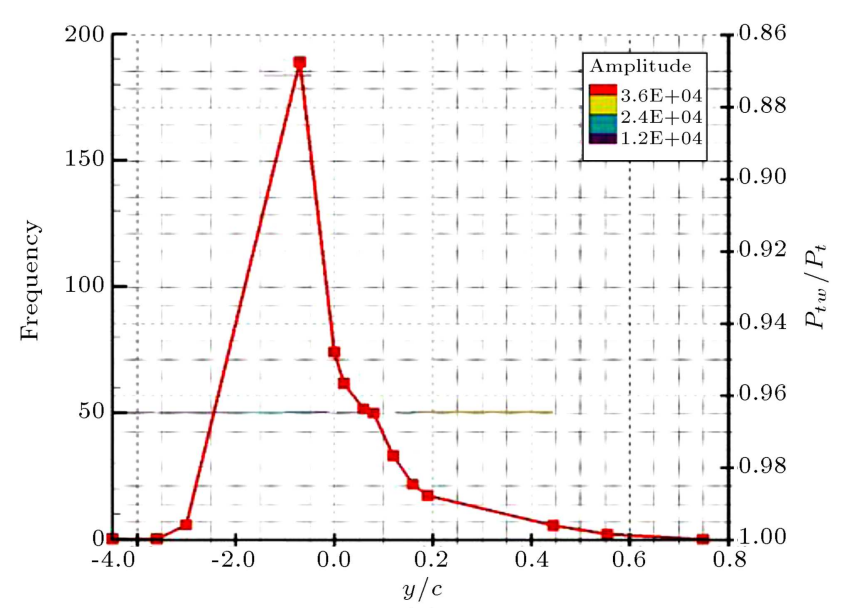

(a)

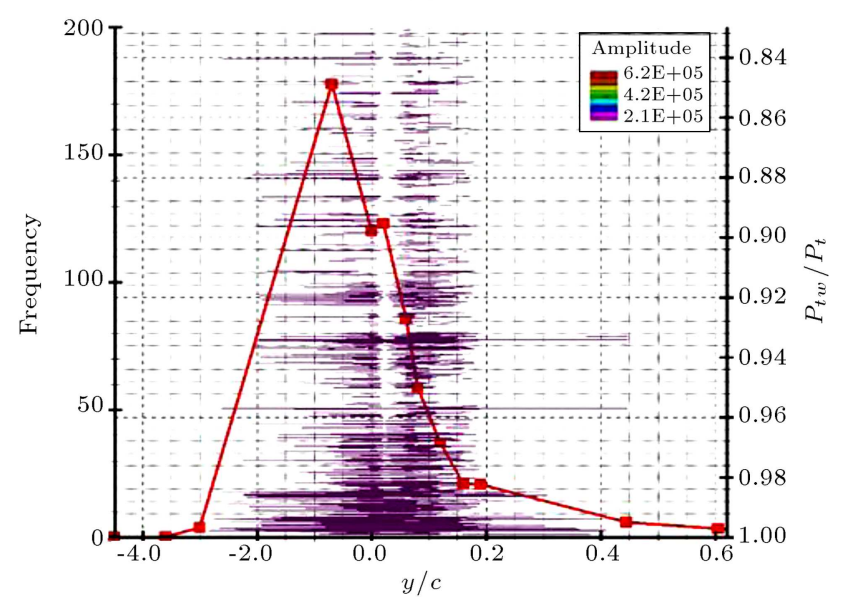

(b)

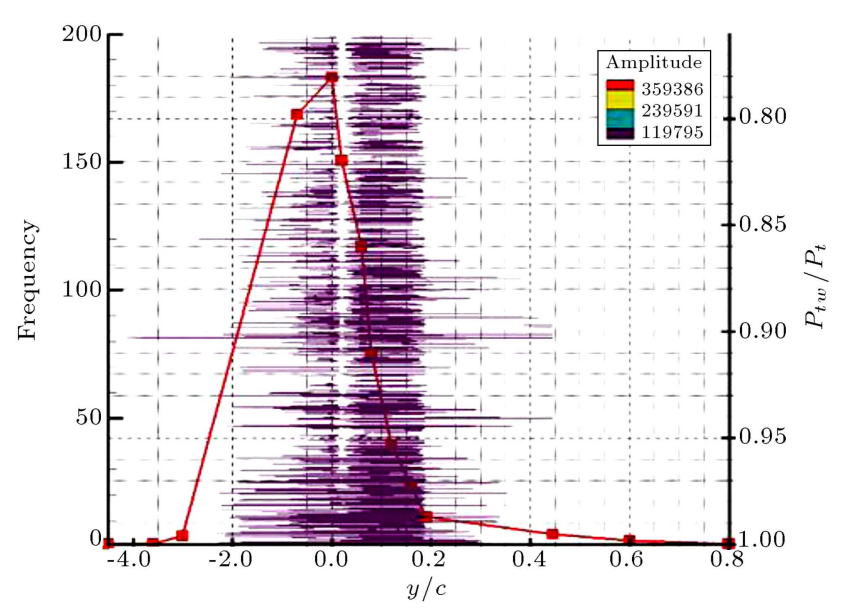

(c)

Figure 15. Wake profile and cross correlation at $M_{\infty}=0.66:$ (a) $\alpha=4.9 \mathrm{deg}$, (b) $\alpha=5.6 \mathrm{deg}$, and (c) $\alpha=6.7 \mathrm{deg}$.

condition is called buffet onset, a condition in which the shock wave oscillates with a certain frequency. The buffet onset was verified through the analysis of the precision error of the sensors on the suction side of the airfoil, where their behavior changed when the shock wave oscillated in their vicinity. Further verification

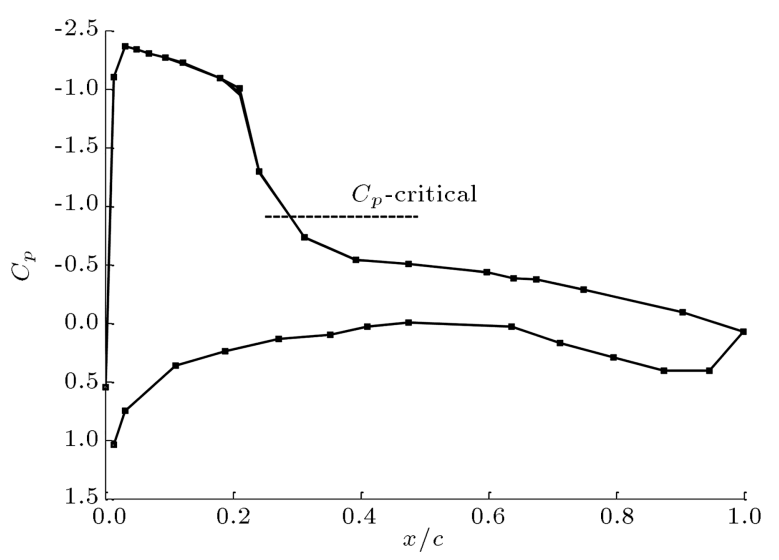

(a)

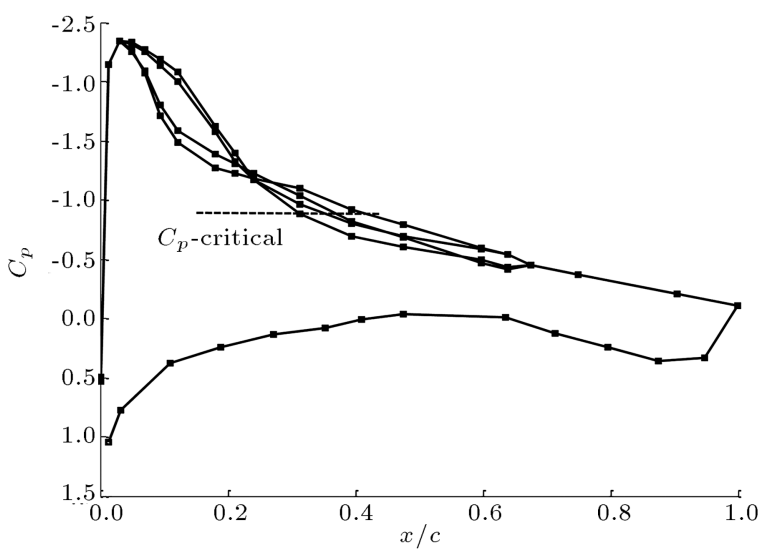

(b)

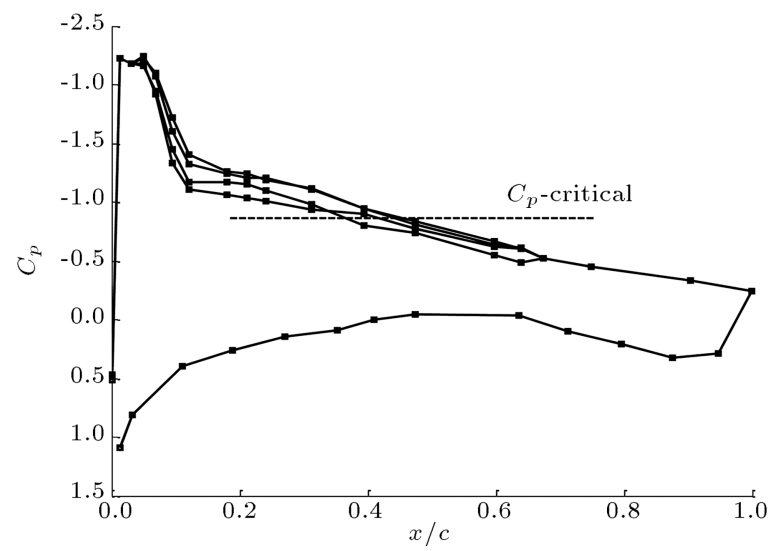

(c)

Figure 16. Pressure fluctuation at $M_{\infty}=0.66$ at different angles of attacks: (a) $\alpha=4.9 \mathrm{deg}$, (b) $\alpha=5.6$ deg, and (c) $\alpha=6.7 \mathrm{deg}$.

was obtained by the analysis of total drag coefficient whose values increased suddenly by measuring the wake profile. From the analysis of the total drag coefficient measured by the rake behind the model, it was observed that the drag divergence was triggered as soon as the buffet onset began. Additionally, Power Spectrum Density (PSD) of the total drag signal showed that it had an oscillation frequency of about $80 \mathrm{~Hz}$. Applying the cross-correlation technique between the reference 
signal at the foot of the shock on the suction side of the model and other signals and repeating this procedure for other sensors in the wake region, this study indicated that the sensors over and behind the model had the same frequency of about $80 \mathrm{~Hz}$ due to shock wave oscillation and growth in the unsteadiness of the flow when buffet phenomenon initiated.

\section{Nomenclature}

$\begin{array}{ll}M_{\infty} & \text { Free Mach number } \\ C_{p} & \text { Pressure coefficient } \\ C_{p, c r} & \text { Critical pressure coefficient (sonic line) } \\ C_{p}^{\prime} & \text { Pressure coefficient precision } \\ \beta & \text { Flap deviation angle } \\ P_{t} & \text { Total pressure } \\ P_{w t} & \text { Total pressure in wake region } \\ \text { PSD } & \text { Power Spectrum Density } \\ \text { FFT } & \text { Fast Fourier Transform } \\ \text { AoA } & \text { Angle of Attack } \\ \alpha & \text { Angle of attack } \\ p & \text { Pressure }\end{array}$

\section{References}

1. Humphreys, M.D., Pressure Pulsations on Rigid Airfoils at Transonic Speeds, NACA Research Memorandum L51I12, National Advisory Committee for Aeronautics (1951).

2. Gao, C., Zhang, W., and Ye, Z. "Reduction of transonic buffet onset for a wing with activated elasticity", Aerospace Science and Technology, 77, pp. 670-676 (2018).

3. Vos, R. and Farokhi, S. "Introduction to transonic aerodynamics", In Fluid Mechanics and Its Applications, 1st Edn, pp. XIII-555, Springer Netherlands, New York London (2015).

4. Lee, B.H.K. "Self-sustained shock oscillations on airfoils at transonic speeds", Progress in Aerospace Sciences, 37, pp. 147-196 (2001).

5. Liu, Y., Wang, G., Zhu, S., and Ye, Z. "Numerical study of transonic shock buffet instability mechanism", 46th AIAA Fluid Dynamics Conference, 13-17 June, Washington, D.C. (2016).

6. Szubert, D., Grossi, F., Garcia, A.J., Hoarau, Y., Hunt, J.C.R., and Braza, M. "Shock-vortex shear-layer interaction in the transonic flow around a supercritical airfoil at high Reynolds number in buffet conditions", Journal of Fluids and Structures, 55, pp. 276-302 (2015).

7. Polentz, P.P., Page, W.A., and Levy, L.L., The Unsteady Normal Force Characteristics of Selected NACA Profiles at High Subsonic Mach Numbers, NACA Research Memorandum A55C02, National Advisory Committee for Aeronautics, May (1955).
8. Mabey, D.G., Some Remarks on Buffeting, Technical Memorandum Structures 980, Royal Aircraft Establishment, February (1981).

9. Sousa, R.S., Girardi, R.D., and Silva, R.G.A. "A new criterion for transonic buffeting onset estimation", 35th AIAA Applied Aerodynamics Conference, AIAA AVIATION Forum, AIAA 2017-4231 (2017).

10. Zhao, Z., Ren, X., Gao, C., Xiong, J., Liu, F., and Luo, S. "Experimental study of shock wave oscillation on SC (2)-0714 airfoil", 51st AIAA Aerospace Sciences Meeting including the New Horizons Forum and Aerospace Exposition, 07-10 January 2013, Grapevine (Dallas/Ft. Worth Region), Texas, AIAA 2013-0537.

11. Fernie, R.M. and Babinsky, H. "Unsteady shock behaviour on a NACA 0012 aerofoil", 41st Aerospace Sciences Meeting and Exhibit, 6-9 January 2003, Reno, Nevada, AIAA 2003-226.

12. Pearcey, H.H. "Some effects of shock-induced separation of turbulent boundary layers in transonic flow past aerofoils", Aeronautical Research Council Reports and Memoranda, Reports and Memoranda No. 3I08 (1959).

13. Lee, B.H.K. "Effects of trailing-edge flap on buffet characteristics of a supercritical airfoil", J. Aircraft, 29(1), pp. 93-100 (1992).

14. Jacquin, L., Molton, P., Deck, S., Maury, B., and Soulevant, D. "Experimental study of shock oscillation over a transonic supercritical profile", AIAA, 47(9), pp. 1985-1994 (2009).

15. Tang, D. and Dowell, E.H. "Experimental aerodynamic response for an oscillating airfoil in buffeting flow", Journal of AIA A, 52(6), pp. 1170-1179 (2014).

16. Kouchi, T., Yamaguchi, S., Koike, S., Nakajima, T., Sato, M., Kanda, H., and Yanase, S. "Wavelet analysis of transonic buffet on a two-dimensional airfoil with vortex generators", Exp Fluids, 57(11), pp. 57-166 (2016).

17. Liu, J. and Zhichung Yang, Z. "Numerical study on transonic shock oscillation suppression and buffet load alleviation for a supercritical airfoil using a microtab", Engineering Applications of Computational Fluid Mechanics, 10, pp. 529-544 (2016).

18. Tian, Y., Gao, S., Liu, P., and Wang, J. "Transonic buffet control research with two types of shock control bump based on RAE2822 airfoil", Chinese Journal of Aeronautics, 30(5), pp. 1681-1696 (2017).

19. Gao, C., Zhang, W., and Ye, Z. "Numerical study on closed-loop control of transonic buffet suppression by trailing edge flap", Computers \& Fluids, 132, pp. 3245 (2016).

20. Golestani, A., Ehghaghi, M.B., and Soltani, M.R. "An experimental study of buffet detection on supercritical airfoils in transonic regime", Journal of Aerospace Engineering, 229(2), pp. 312-322 (2015).

21. Golestani, A., Soltani, M.R., and Masdari, M. "Effect of wind tunnel porosity on the flow around an oscillating airfoil at transonic speed", Scientia Iranica, 24(3), pp. 1069-1076 (2017). 
22. Amiri, K., Soltani, M.R., and Haghiri, A. "Steady flow quality assessment of a modified transonic wind tunnel", Scientia Iranica, 20(3), pp. 500-507 (2013).

\section{Biographies}

Mehran Masdari is an Assistant Professor at the New Sciences and Technologies Department in University of Tehran. He has a PhD degree in Aerospace Engineering from the Sharif University of Technology in 2011. His research interests are applied aerodynamics, wind tunnel testing, neural network, and data processing.

Masoud Talebi received his MS degree in Aerospace Engineering from Sharif University of Technology in 2013. His research interests are wind tunnel testing.

Aqil Zeinalzadeh received his MS degree in Aeros- pace Engineering from Sharif University of Technology in 2014. His research interests are wind tunnel testing, unsteady aerodynamics, applied aerodynamics, and renewable energy.

Mohammad Ali Abdi received his MS degree in Aerospace Engineering from Sharif University of Technology in 2013. His research interests include unsteady aerodynamics, wind tunnel testing, applied aerodynamics, and renewable energy.

Mohammad Reza Soltani is a Professor at the Aerospace Engineering Department of Sharif University of Technology, Tehran, Iran. He has a PhD degree in Aerodynamics from the University of Illinois at Urbana-Champaign, USA. His research interests include applied aerodynamics, unsteady aerodynamics wind tunnel testing, wind tunnel design, and data processing. 